Elvin M. Aliyev

DOI: 10.25045/jpit.v07.i2.07

NASA Institute for Space Research of Natural Resources, Baku, Azerbaijan

elvin.aliyev.m@gmail.com

\title{
TRANSFORMATION AND INTERPOLATION ISSUES IN GEO-INFORMATION MODELING
}

Data geo-reference and interpolation issues are the main problems faced while creating the geoinformation model. The article reviews the transformation of the raster maps of different scales, and explains existing methods within the geo-reference operations. Numerous deterministic and geo-statistic interpolation methods are presented. Moreover, LIDAR and GeoEye-1 data analysis is conducted for the geo-information modeling.

Keywords: Lagrange polynomials, interpolation, LIDAR, GeoEye-1, Affine, Polynomial, ArcGIS, transformation, geo-reference, IDW, ordinary kriging.

\section{Purpose and relevance of the study}

The main issues, occurring in the projects that implemented within the geo-information systems, are usually related to geo-reference operations. Moreover, it is necessary to interpolate the spatial information, which is important for the modeling of geo-information.

The geo-information modeling of objects and events includes several phases. These stages consist of the studied objects and events to have accurate attribute information and of their spatial relations. Reflecting particular features of the object and event, such a model enables effective results when reviewing and forecasting the current situation. In this case, the studied object and event may not exist in reality. The most interesting moment when developing geo-information models related to a particular form of presentation is not which time interval the studied object and event are attributed to, but providing a more accurate description of their image and properties in reality. Therefore, the world, objects and events that surround us is a complex system. Sometimes any process, as well as all parts of events going on in this complex and unique system cannot be studied. These models have the properties as to reflect the current situation, as well as to forecast. For this purpose, various modern approaches, methods and algorithms are available. Geo-information models also rely on modern approaches and their special features is the fact that they have an opportunity to give more information about the object and event. The reason for this is that the information about graphical and text data is concentrated in these models. One of the advantages of geo-information models is that they are connected to respective coordinate systems and cartographic projections, as well as building up triangulation networks according to the known points. For this reason, transformation and interpolation of the spatial data is of great importance among the factors that contribute to the creation of geographic information model and is considered urgent.

Georeference operation includes the image scaling, defining the rotation angle, residue and other errors and other procedures. These procedures requires the application of the Affine or Polynomial methods depending on the image features and transformed shapes. To that end, the unknown coefficients are determined based on georeference, and then, these methods should be reviewed in geo-information systems. For this reason, georeference methods should be studied and the transformation methods to be applied for different scaled images should be identified. The study of these methods and a comparative analysis of existing methods may contribute to the achievements of effective results in programming issues. It is known that modeling of objects and events in geo-information systems is solved through the application of various mathematical methods. Typically, differences or similarities are observed in the results of the models, which proves the availability of numerous approaches and methods in these issues.

The paper examines Affine and Polynomial transformations of raster data and reviews mean squared error calculation methods in accordance with the general image, as well as with the coordinates $x, y$ of each point and analyzes geo-statistic and other interpolation methods. Explains existing methods 
within geo-reference operations. Moreover, LIDAR data based TIN (Triangulated irregular network) modeling and other arisen issues are presented.

\section{Problem solution techniques}

Geo-information modeling accuracy depends on the georeference of raster data a lot. It is due to the fact that the image is closely connected to the geographic coordinate system and cartographic projection during the georeferenced operation, which enables exact calculations based on it and creates a more accurate model.

Raster images are often obtained by scanning of paper maps, aerospace data processing and other operations. As the scanned images have no spatial connections, for their involvement in the georeference operation, in most cases, spatial information connected to the coordinate system is used. The spatial information used for the connection may have point, areal, and linear properties. Such information is also called reference objects. Reference objects are used for the establishment of Polynomial transformations so they could carry out the spatial determination of raster images. Note that during the georeference connection of the image should equally be carried out. So, the image connection methods vary depending on the scale reduction. Experience shows that the transformation should be implemented both on the image corner and center to ensure the accuracy georeferenced operation. In order to minimize the errors during such operations, it is important to use non-deformed images, the resolution of which is high. In this case, dpi (dots per inch) indicators used for the raster image formation should also be considered. Thus, the scanning quality depends on the number of points within $1 \mathrm{inch}$. Reviewed issues require high dpi and the use of high-performance computers for image processing.

Large format scanners are used to perform image electronization during the mapping. To ensure more accurate transactions, at least600 dpi is required [1]. The image scanning with high resolution allows maintaining its natural color. However, it also should be noted that the higher resolution of the image is, the larger the size is [2]. For this reason, for the high quality image, it is appropriate to scan small-scale maps (paper maps) with high-dpi performance.

The article deals with GeoEye-1 data georeferenced issues and uses polynomial-2 method for this purpose (Figure 1).

During Raster georeference, Affine (linear) method defines the transformation as 6 parameters

$$
\begin{aligned}
& u=a_{0}+a_{1}{ }^{x}+a_{2}{ }^{y} \\
& v=b_{0}+b_{1}{ }^{x}+b_{2}{ }^{y}
\end{aligned}
$$

Here, $(x, y)$-denote the pixel coordinates of the coordinate system; $(u, v)$ - pixel coordinates found after transformation; $\left(a_{0-2}, b_{0-2}\right)$ - transformation coefficients.

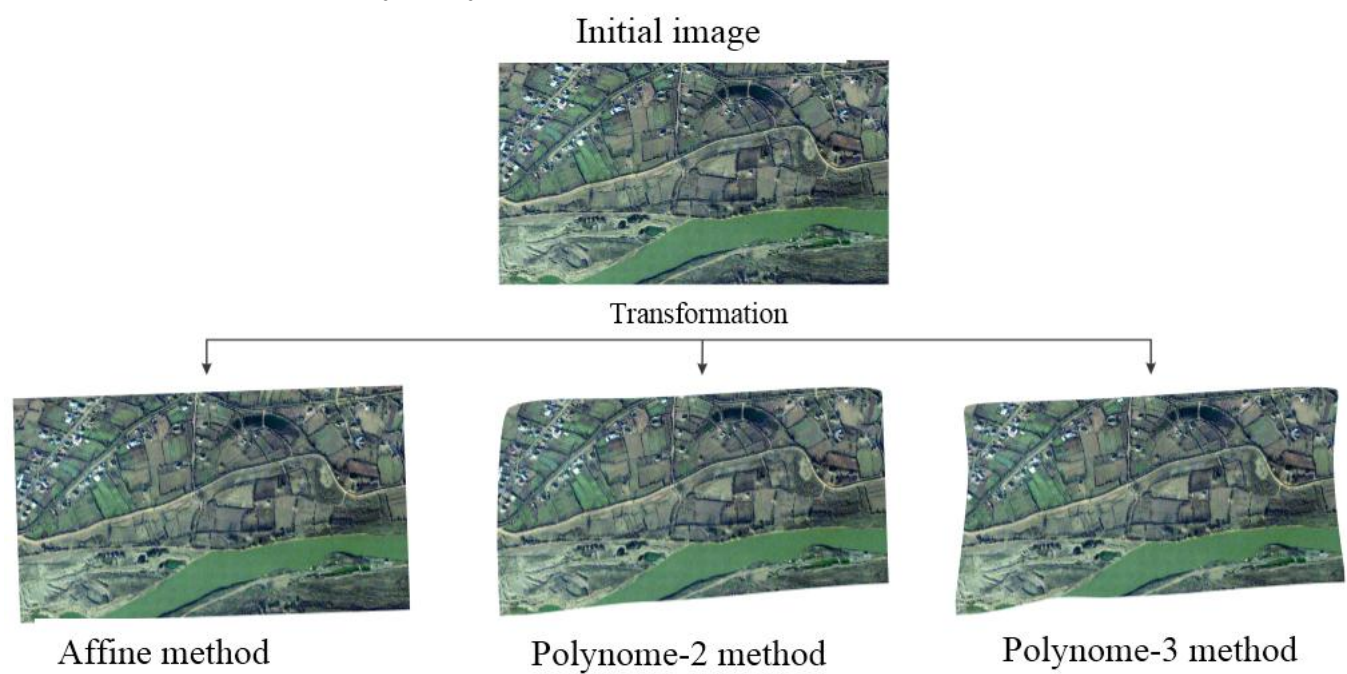

Figure 1. Transformation of the initial image through Affine and polynomial methods 
However, the majority of the reference points obtained from the georeferenced urges the application of polynomial-2 method (Figure 2). It should be noted that the scale of the image also enables the application of this method. In the case of polynomial-2 method, system of equations can be expressed as follows:

$$
\begin{gathered}
u=a_{0}+a_{1}^{x}+a_{2} y+a_{3} x y+a_{4} x^{2}+a_{5} y^{2} \\
v=b_{0}+b_{1}^{x}+b_{2} y+b_{3} x y+b_{4} x^{2}+b_{5} y^{2}
\end{gathered}
$$

Where $(u, v)$-denotes the pixel coordinates found after the transformation; $\left(a_{0}, b_{0}\right)$ - image shift coefficients on $x$ and $y$-axes; $\left(a_{1}, a_{2}, b_{1}, b_{2}\right)$ - linear change coefficients of the scale on $\mathrm{x}$ and $\mathrm{y}$ axes; $(x, y)$ - pixel coordinates in the original coordinate system; $\left(a_{3}, b_{3}\right)$ - value of the rotation angle of the image; $\left(a_{4}, a_{5}, b_{4}, b_{5}\right)$ - non-linear change of the scale.

Thus, for calculating the image coordinates knowing the coefficients of the polynomials, the system of equations of ArcGIS software (2) is used. As the other methods available in the same platform are required during the transformation of the rasters exposed to high distortion and deformation, their use are not considered appropriate. With the introduction of polynomial-2, themean squared error over the raster is estimated in $0.24 \mathrm{~m}$.

After determining the reference objects, the raster data can be connected to the coordinate system with the use of polynomial or spline methods. In non-linear methods, polynomial-2 and its top methods are often used. Polynomial-3 is applied for the connection of the maps deformed during scanning. Polynomial-4 is applied in a very few cases and used for aerial-photos with high distortion [3]. The choice of terrestrial control points play an important role in assessing transformation coefficients. In

\begin{tabular}{|c|c|c|c|c|c|}
\hline Link & X Source & Y Source & X Map & Y Map & Residual \\
\hline 1 & 275026,556767 & 4426516,667360 & 275026,547242 & 4426516,662421 & 0,08922 \\
\hline 2 & 274978,527906 & 4425966,081461 & 274978,515074 & 4425966,079829 & 0,00929 \\
\hline 3 & 273918,377257 & 4425994,389042 & 273918,370377 & 4425994,394128 & 0,03728 \\
\hline 4 & 273911,481313 & 4426525,458495 & 273911,484527 & 4426525,454179 & 0,01105 \\
\hline 5 & 274506,902777 & 4426305,433775 & 274506,737804 & 4426305,114030 & 0,62330 \\
\hline 6 & 274624,650581 & 4426342,518480 & 274625,064668 & 4426342,978406 & 0,35225 \\
\hline 7 & 274624,498999 & 4426342,327904 & 274624,892689 & 4426342,740280 & 0,30411 \\
\hline 8 & 274624,387613 & 4426342,135942 & 274624,575188 & 4426342,356634 & 0,04400 \\
\hline 9 & 274624,166681 & 4426341,864491 & 274624,363521 & 4426342,025904 & 0,01504 \\
\hline 10 & 274572,677864 & 4426496,032337 & 274576,252474 & 4426498,381297 & 0,00036 \\
\hline 1 & & & I'I & & 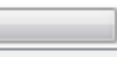 \\
\hline \multicolumn{2}{|c|}{ V Auto Adjust } & Transformation: & \multirow{2}{*}{\multicolumn{2}{|c|}{ 2nd Order Polynomial }} & \\
\hline \multicolumn{2}{|c|}{ Total RMS Error: } & 0,24835 & & & \\
\hline
\end{tabular}
this case, the transformation accuracy is closely related to the election accuracy of the control points.

Figure 2. Image georeference results through polynomial - 2

It should be noted that the linear transformations are considered to be the simplest method and called polynomial-1 (Affine method). In such transformations, unprocessed raster should be reduced to the cartographic projection plane of the image. This method is mainly used for transforming largescaled maps. It should be noted that linear transformation matrix includes the determination of 6 coefficients [4]. The higher the degree of Polynomials is, the more the complex distortions over the image can be adjusted just. Thus, the urgency of implementing the top points of the Polynomial arises from the majority of the reference points [5] and it is required for small-scaled mapping.

As a rule, during the transformation of the images of different scales their compression 
characteristics is not taken into consideration. Irrespective of the scales, any core data loss should be avoided during the mapping and geo-information analysis.

During compression, the images are stored in such a way that their initial condition cannot be restored and the data completely loses its original condition [6]. For this reason, working with raster data the use of compression operations is not recommended.

There are different types of raster formats for image storage, and they are the formats without data loss (TIFF, BMP), as well as with the data loss (JPEG, GIF). It should be noted that the common format for storing aerospace images are not available. However, there are common structural elements of the formats of multispectral space images submitted by the terrestrial receiving stations and initial processing centers. For example, the service information about the image (e.g., date and time of shooting, shooting apparatus type, height, calibration data, pre-processing information etc.). Compression of digital images includes fitting and reduction of graphical information in terms of volume, which is provided in bits or bytes. This process is required for saving memory capacity, which is required to write and store the images during the data transmission through the small satellites channels to the Earth. The compression process is possible in two cases - without or with data loss. If there are objects of the same tones (clean water basins etc.) in any image, in this case, the information is not lost during the compression and brightness values are replaced by a single value. Methodically, in this kind of the compression, the information volume of the aerospace images is reduced twice averagely, but the image is completely restored and the issue is resolved through the use of decompression method. The decompression does not completely restore the image details [7].

The input data of geo-information systems may contain topographic maps and plans, aerospace data, and terrestrial observation results. In geo-information systems platform, transformation issues are often implemented the following methods and the coordinate system is set up for it. The first system is related to the original image (untransformed image). In this system, if we denote the point coordinates by $\left(X_{\text {old }}, Y_{\text {old }}\right)$, and denote the point coordinates of the second system (the image after the transformation) by $\left(X_{n e w}, Y_{n e w}\right)$, the two points scaling, rotation angle determination, and the determination of the transposition coefficient can be performed with the following method (a special case of Affine transformation) [ 8]:

$$
\left.\begin{array}{l}
X_{\text {new }}=k\left(X_{\text {old }}-X_{0}\right) \cos \theta+\left(Y_{\text {old }}-Y_{0}\right) \sin \theta ; \\
Y_{\text {new }}=k\left[-\left(X_{\text {old }}-X_{0}\right)\right] \sin \theta+\left(Y_{\text {old }}-Y_{0}\right) \cos \theta,
\end{array}\right\}
$$

Here, $\left(X_{0}, Y_{0}\right)$ - is transposition value of coordinate origin of the second system in relation to the coordinate origin of the first system; $\theta$-rotation angle; $k$-scaling coefficient.

Raster transformations through Polynomial-5 method are defined as follows:

$$
\left.\begin{array}{l}
X_{\text {new }}=a_{1} X_{\text {old }}^{5}+a_{2} Y_{\text {old }}^{5}+a_{3} X_{\text {old }}^{4} Y_{\text {old }}+a_{4} X_{\text {old }}^{4} Y_{\text {old }}^{4}+a_{5} X_{\text {old }}^{3} Y_{\text {old }}^{2}+a_{6} X_{\text {old }}^{2} Y_{\text {old }}^{3}+a_{7} Y_{\text {old }}^{4}+ \\
a_{8} Y_{\text {old }}^{4}+a_{9} X_{\text {old }}^{3} Y_{\text {old }}+a_{10} X_{\text {old }} Y_{\text {old }}^{3}+a_{11} X_{\text {old }}^{2} Y_{\text {old }}^{2}+a_{12} X_{\text {old }}^{3}+a_{13} Y_{\text {old }}^{3}+a_{14} X_{\text {old }}^{2} Y_{\text {old }}+ \\
+a_{15} X_{\text {old }} Y_{\text {old }}^{2}+a_{16} X_{\text {old }}^{2}+a_{17} Y_{\text {old }}^{2}+a_{18} X_{\text {old }} Y_{\text {old }}+a_{19} X_{\text {old }}+a_{20} Y_{\text {old }}+a_{21} ; \\
Y_{\text {new }}=a_{22} X_{\text {old }}^{5}+a_{23} Y_{\text {old }}^{5}+a_{24} X_{\text {old }}^{4} Y_{\text {old }}+a_{25} X_{\text {old }} Y_{\text {old }}^{4}+a_{26} X_{\text {old }}^{3} Y_{\text {old }}^{2}+a_{27} X_{\text {old }}^{2} Y_{\text {old }}^{3}+ \\
+a_{28} X_{\text {old }}^{4}+a_{29} Y_{\text {old }}^{4}+a_{30} X_{\text {old }}^{3} Y_{\text {old }}+a_{31} X_{\text {old }} Y_{\text {old }}^{3}+a_{32} X^{2}{ }_{\text {old }} Y_{\text {old }}^{2}+a_{33} X_{\text {old }}^{3}+a_{34} Y_{\text {old }}^{3}+ \\
+a_{35} X_{\text {old }}^{2} Y_{\text {old }}+a_{36} X_{\text {old }} Y_{\text {old }}^{2}+a_{37} X_{\text {old }}^{2}+a_{38} Y_{\text {old }}^{2}+a_{39} X_{\text {old }} Y_{\text {old }}+a_{40} X_{\text {old }}+a_{41} Y_{\text {old }}+a_{42}
\end{array}\right\}
$$

Here, $a_{1}, a_{2}, \ldots a_{42}$ are the transformation coefficients.

The final values results obtained from the application of the polynomial methods depend on the reference objects, and in this case, the calculation of the mean squared errors is required both by general raster and by each reference point.

The conducted research proves that regardless of the scale, georeference of any deformed image does not provide efficient results. The use of deformed image is generally not recommended 
in various projecting, engineering issues, especially in the legal registration of lands and properties. In some cases, paper maps and plans are need to be georeferenced. It should be noted that the errors arising from the raster georeference tend to have different values. These values require the application of the top polynomial methods depending on the number of reference objects.

The studies show that reference points with the known coordinates and digital terrain models should be used for more accurate implementation of the reviewed issue [9]. Image processing process includes its transformation, creation of mosaics, and etc. As mentioned, the coefficients of the image scale, angle and other factors should be set during the image transformation. However, for mountainous areas ortho-transformation is used [10]. Ortho-transformation process is the transformation of an image into an orthogonal projection plane and eliminates the relief-based distortions.

At present, orthophotoplans are more widely used in the preparation of maps and plans, as well as in projecting works, and they are included to more informative data used as a geographical basis.

It should be noted that, in any case, all rates of polynomial model can be applied to the spatial connection of cadastral maps. Nevertheless, in this case, the residual and root mean square error (RMSE) must be considered [11]. Approaching to each point, individually mean square errors by each point as per the residual errors are defined as follows:

$$
R x=\sqrt{\frac{1}{n} \sum_{i=1}^{n} X R_{i}^{2}} \quad(5) ; \quad R y=\sqrt{\frac{1}{n} \sum_{i=1}^{n} Y R_{i}^{2}} \quad(6) ; \quad T=\sqrt{X R_{i}^{2}+Y R_{i}^{2}}
$$

Here $R x, R y$-denotes the overall mean square errors by $x$ and $y$ axes; $T$-overall mean square error; $n$ - total number of reference objects; $X R_{i}$ and $Y R_{i}$ - values of the $i$-indexed residual errors by $x$ and $y$ axes [12].

Studying the solution methods of various mathematical problems and developing algorithms, based on them, are of utmost importance for modeling of objects and events.

Generally, in the exploration of any process, based on computational mathematics, several stages should be noted. In initial phases, the mathematical model is chosen, that is, algebraic, differential or integral equations are used for the description of the process. More precisely, for the process description, it should be expressed in the form of a certain mathematical structure. Then, the problem solution is set with approximate numerical method, i.e., the calculation algorithm is selected. The following steps consist of the algorithm programming and the calculations based on the application [13].

Obviously, a function is provided in various ways. One of them is the schedule method: the function is provided as a finite number of a variable $(x)$, i.e., $f\left(x_{i}\right), i=0,1, \ldots, n$. However, in practice, the function value sometimes is required to be calculated with other values of the variable. In this case, a simple function $P(x)$ is set so that the condition

$$
P\left(x_{i}\right)=f\left(x_{i}\right), \quad(i=0,1, \ldots, n)
$$

to be provided, and $P(x)-f(x)$ to be poor enough in other points of determination field of $f(x)$. Setting such function $P(x)$ is called an interpolation. Function $P(x)$ is usually found in the polynomial form . Sometimes, the function is given in an analytical form, but finding its value at the arbitrary point of determination field requires high computations. In this case, interpolation is used. Using the analytical expression of the function, its value is found at several points that are easy to be calculated, and the polynomial $P(x)$ is set using these values [14].

If the experimental data is given within $[a, b]$ and it is required to be expressed by any function $L(x)$ within, then, an approximation is called an interpolation [15].

The article explores the Parabolic and Lagrange, IDW (inverse distance weighting), as well as ordinary Kriqinq methods of interpolation. 
According to the parabolic interpolation method, in which the function $y=f(x)$ provided as a table is required to be replaced by a such polynomial $L(x)$ that the values of this function at distribution points will be equal to the table value of the function $f(x)$, i.e.,

$$
L\left(x_{0}\right)=f\left(x_{0}\right)=y_{0}, L\left(x_{1}\right)=f\left(x_{1}\right)=y_{1}, \ldots, L\left(x_{n}\right)=f\left(x_{n}\right)=y_{n}
$$

According to this method, approximating function $L_{n}(x)$ is foundas follows:

$$
L_{n}(x)=a_{0}+a_{1} x+a_{2} x^{2} \ldots+a_{n} x^{n}
$$

Where $a_{0}, a_{1}, a_{2}, \ldots, a_{n}$ are the constant coefficients and found under the conditions (8). To this end, the equation of the following system is compiled. The main condition here is writing the expression (9) for each distribution point, i.e., for $\left(x_{0}, y_{0}\right),\left(x_{1}, y_{1}\right),\left(x_{2}, y_{2}\right), \ldots,\left(x_{n}, y_{n}\right)$.

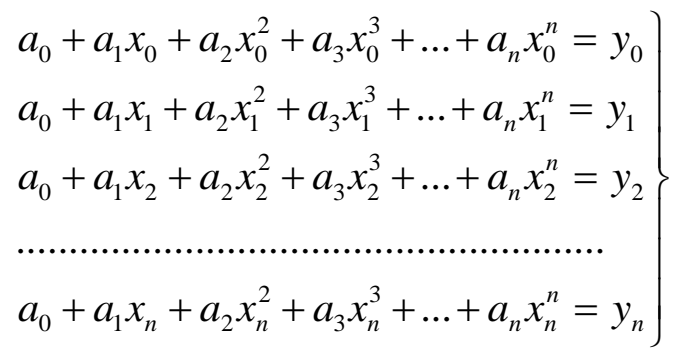

Where $x_{0}, x_{1}, x_{2}, \ldots, x_{n}, y_{0}, y_{1}, y_{2}, \ldots, y_{n}$ are the argument and function values.

Lagrange interpolation method is as follows:

$$
L_{n}(x)=\sum_{i=0}^{n} \frac{\left(x-x_{0}\right)\left(x-x_{1}\right) \ldots\left(x-x_{i-1}\right)\left(x-x_{i+1}\right) \ldots\left(x-x_{n}\right)}{\left(x_{i}-x_{0}\right)\left(x_{i}-x_{1}\right) \ldots\left(x_{i}-x_{2}\right)\left(x_{i}-x_{i+1}\right) \ldots\left(x_{i}-x_{n}\right)} y_{i}
$$

Depending on the polynomial degree $(n=1,2)$, interpolated polynomials are expressed as follows:

$$
\begin{gathered}
L_{1}(x)=\frac{x-x_{1}}{x_{0}-x_{1}} y_{0}+\frac{x-x_{0}}{x_{1}-x_{0}} y_{1}, n=1 \\
L_{2}(x)=\frac{\left(x-x_{1}\right)\left(x-x_{2}\right)}{\left(x_{0}-x_{1}\right)\left(x_{0}-x_{2}\right)} y_{0}+\frac{\left(x-x_{0}\right)\left(x-x_{2}\right)}{\left(x_{1}-x_{0}\right)\left(x_{1}-x_{2}\right)} y_{1}+\frac{\left(x-x_{0}\right)\left(x-x_{1}\right)}{\left(x_{2}-x_{0}\right)\left(x_{2}-x_{1}\right)} y_{2} \cdot n=2
\end{gathered}
$$

When the interpolation function is $n=1$, it is linear, and if $n=2$, it is parabolic [16]. However, when $n=3$, it is expressed as follows [17].

$$
\begin{aligned}
& L_{3}(x)=\frac{\left(x-x_{1}\right)\left(x-x_{2}\right)\left(x-x_{3}\right)}{\left(x_{0}-x_{1}\right)\left(x_{0}-x_{2}\right)\left(x_{0}-x_{3}\right)} y_{0}+\frac{\left(x-x_{0}\right)\left(x-x_{2}\right)\left(x-x_{3}\right)}{\left(x_{1}-x_{0}\right)\left(x_{1}-x_{2}\right)\left(x_{1}-x_{3}\right)} y_{1}+ \\
& +\frac{\left(x-x_{0}\right)\left(x-x_{1}\right)\left(x-x_{3}\right)}{\left(x_{2}-x_{0}\right)\left(x_{2}-x_{1}\right)\left(x_{2}-x_{3}\right)} y_{2}+\frac{\left(x-x_{0}\right)\left(x-x_{1}\right)\left(x-x_{2}\right)}{\left(x_{3}-x_{0}\right)\left(x_{3}-x_{1}\right)\left(x_{3}-x_{2}\right)} y_{3} .
\end{aligned}
$$

For the interpolation through the ordinary Kriging, the following expression is used [18].

$$
Z(s)=\mu+\varepsilon(s)
$$

Here, $s=(X, Y)$-denotes the coordinates of the point; $Z(s)$ - identic indicator of the point; $\mu$-constant mean indicator; $\varepsilon(s)$ - random errors related to spatial relations.

Using the methods of IDW, the calculations are carried out in the following way:

$$
\hat{Z}\left(s_{0}\right)=\sum_{i=1}^{N} \lambda_{i} Z\left(s_{i}\right)
$$


Here, $\hat{Z}\left(s_{0}\right)$ - denotes the indicator sought for the point $s_{0} ; N$ - number of reference points; $\lambda_{i}$ - weight of each point used in the model; $Z\left(s_{i}\right)$ - indicator measured at the point $s_{i}$ [19].

LIDAR data modeling and interpolation are reviewed in the case study. To this end, the images are combined at the initial phase, and then, modeling is implemented. Being consisted of two parts, the data restricts geo-information analysis of the image (Figure 3). As a result of the LIDAR data combination, the image could maintain the properties of its original condition. To resolve the problem, based on ArcGIS the images, are combined by following the procedures as Data Management Tools - Raster - Raster Dataset - Mosaic To New Raster.

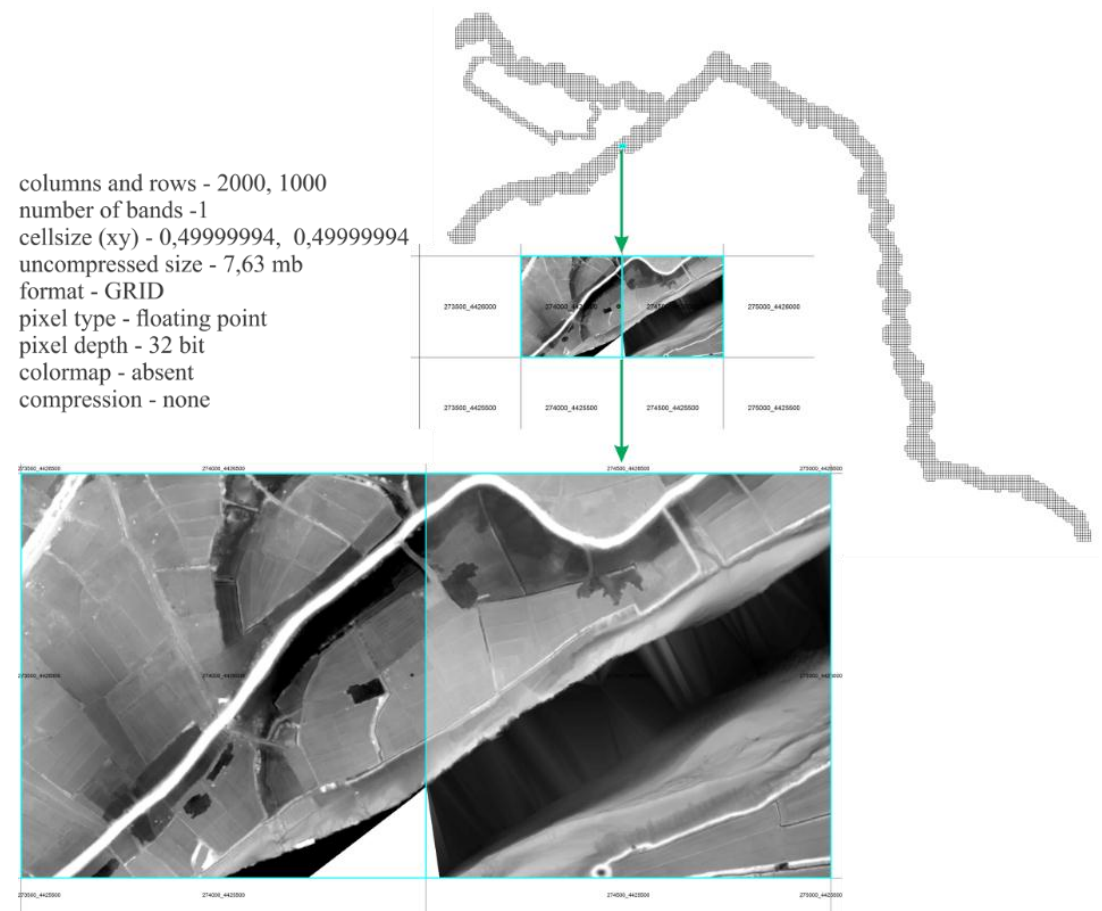

Figure 3. Presentation of LIDAR data (shot of 2010) in UTM 39 projection on the research area

Subsequently, based on LIDAR data, the irregular triangulation network of the flooding area is developed, and the height points are obtained from the density points of the area. The multiple points complicate their processing by means of application, and interpolation is carried out to solve the problem (Figure 4). After the Spatial Analyst based interpolation TIN (triangulated irregular network) is built and site-altitude model is developed (Figure 5) [20]. It can b noted that there is different modeling software and their advantage is to implement the pricing used in these studies on coordinate systems. One of the main problems encountered in modeling is vague description of the interpolation, i.e., describing the dots under sharp angle when developing TIN model. It can be corrected through the visualization. Spline interpolation method is more widely-used in developing non-fuzzy curved lines.

The article reviews raster geo-reference methods in order to increase the precision of geoinformation modeling, and comments on interpolation methods according to various terms and conditions. It is known that geo-information analysis methods of modeling use various deterministic and geo-statistic interpolation methods are used. Complex research of such methods is of great importance for modeling issues of various themes [21].

It should be noted that interpolation of geo-information systems differs from abovementioned methods. In geo-information systems, the points to be interpolated are located on the proper coordinate system, in which the address of each point is known, and any line can pass through the points with a single value based on particular surveys. Raster interpolation is also based on this principle. TIN and high-sensitivity models are also interpolated with the use of this principle. This principle is referred to the geo-statistic analysis methods. However, the 
identification value here, the distances between the dots, and other factors are taken into account. They are classified according to different criteria, and these methods are mentioned above.

a

b

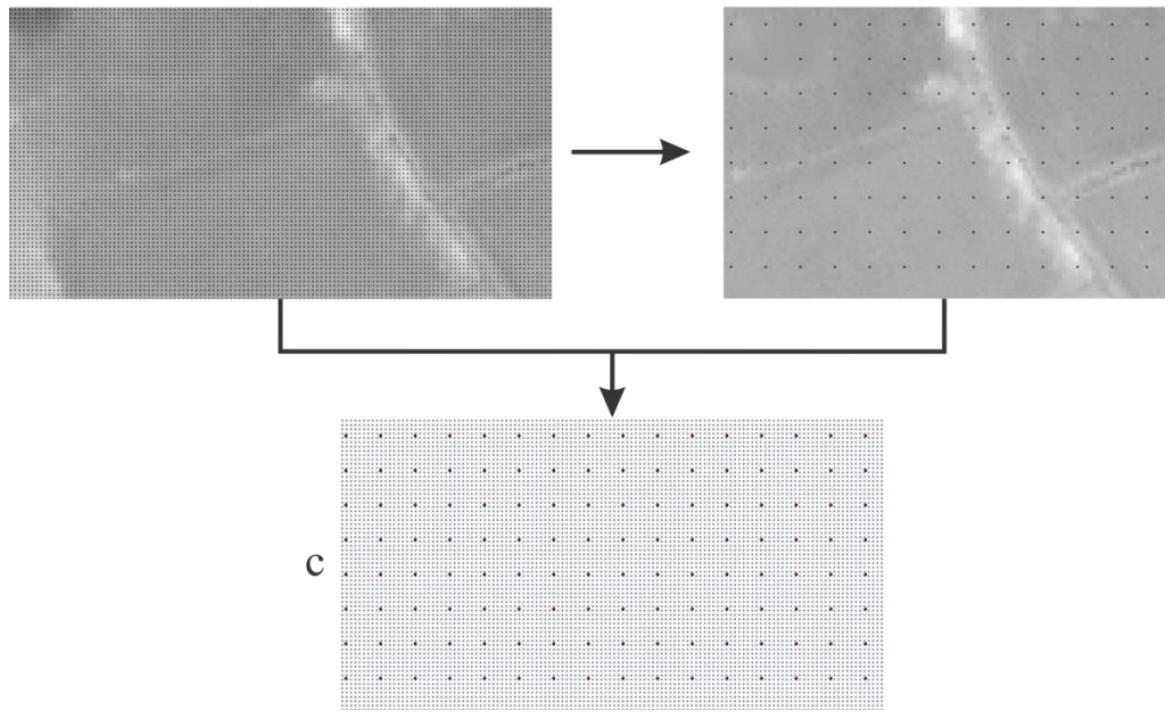

Figure 4. Position of the points to be interpolated over the raster as an example of the research area (a- position of the densely distributed height points over the raster, b-bringing the height points to the tenuous position, c- overall position of the dense and sparse height points).

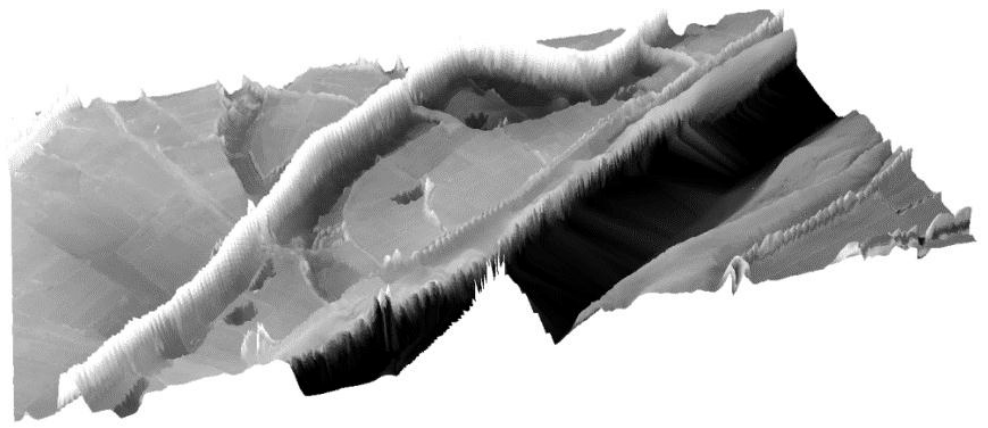

Figure 5. LIDAR data based 3D model of the research area

Experience shows that the geo-information modeling is widely applied in environmental protection, control of emergency situations, legal registration of real estate, cadastral issues, management of municipal and agricultural lands, ecological issues, and other areas. It should be noted that in our country, the necessary steps have been taken in this area. Hence, [22] explores the mapping of soil-plant facilities and roads, [23] reviews object-oriented classification of soil, and [24] highlights the component relations' modeling relations based on the natural component maps. Geo-information systems are widely used in the management of emergency situations of natural origin and in the classification of active areas of real estate market [25-27], in the study of the turbidity in the river of the Middle Kura depression, and soil and vegetation of the south slope of the Greater Caucasus Mountains [28-29], and in the digital mapping for regional planning [30]. The application of geo-information systems in the study of the topography and landscape of the country is also of great importance. Hence, [31] developed digital models of land cadastre plans based on AutoCAD and ArcGIS, [32] conducts geo-information analysis in the real estate, and [33] develops the models for morphometric analysis of the landscape. Moreover, electronic maps of Shamakhi and Ismayilli regions are drawn to study the erosion processes [34], and a model of tourism routes is established to examine the possibility of ecotourism in the region [35]. At the 
same time, local experts have carried out research on developing landscape plans [36] and various thematic maps, as well as in the study of biological diversity [37].

A wide application of GIS in various fields in a short period is due to its positive results in the study of objects and events. Fortunately, the Republic of Azerbaijan has made some progress in this area.

\section{Conclusion}

Information support of geo-information model directly depends on the geometric and other factors of the spatial data available in the geo-database. It should be noted that the exact geoinformation models, based on the complex approaches, are of particular importance in many areas. The application of geo-information models is very topical in urban and regional planning, establishment of control systems (agricultural lands control system, etc.), in forecasting emergency situations and other processes.. The information support of the model also significantly depends on its geographical base and on the resolution, under which it is interpolated. There are various methods to ensure the accurate implementation of these operations, and respectively, these methods should be studied in accordance with the specific terms and conditions set forth from the application point of view. For this reason, the article highlights the transformation and interpolation methods pertained to the development of these models.

Accordingly, the article reviews the image geo-reference issues, Affine and top Polynomial methods; multispectral images are geo-referenced through polynomial-2 method, and the evaluation methods of mean square errors through ArcGIS application are explored. Furthermore, the article highlights the methods of calculating higher rates of polynomial models. The methods of calculating mean square error of the point by the raster are explained.

The article also interprets interpolation methods, the combination of multi-fragment images, and the development of TIN models.

\section{References}

1. www.graphtec.ru/application/cartography/

2. skanworld.ru/razreshenie-skanera-kakoe-znachenie-optimalnoe.html

3. Tokareva O.S. Processing and interpretation of remote data sensing, Tomsk: Publishing house of Tomsk Polytechnic University, 2010, 148 p.

4. Methodical guidelines for implementation of student work on Photogrammetry and Remote Sensing. Comp. Lisitsyn V.E. Kharkov: KNAME 2006, 90 p.

5. gis-lab.info/qa/polynom.html

6. www.ukrprint.com/prepress/theory/img_compress.php

7. Aliyev E.M. MZ data storage, processing and compression issues in different enhancements / proceedings of third scientific-practical conference "The prospects of geodesy and cartography in Azerbaijan", Baku: Ulu, 2011, pp.191-199.

8. Kapralov E.G., Koshkarev A.V., Tikunov V.S., et all. Basics of Geoinformatics:. 2 books. Book 1: M.: Publishing Center "Academy”, 2004, 352 p.

9. www.scanex.ru/ru/publications/pdf/publication21.pdf

10. Labutina I.A., Baldin E.A., The use of remote sensing for ecosystems monitoring of protected areas. Methodical manual, M .: WWF Russia, 2011, 88 p.

11. Maria A. Brovelli, Marco Minghini. Georeferencing old maps: a polynomial-based approach for Como historical cadasters // e-Perimetron, 2012, vol.7, no.3, pp.97-110.

12. www.gis.usu.edu/ doug/rs5750/lectures/L7_Geometriccorr.pdf

13. Huseynov Z.G. Calculation methods and practice. Textbook for high schools, Baku, "Adiloghlu" Publishing House, 2003, 456 p.

14. Mammadov Y.J. Approximate calculation methods, "Baku University" Publishing House, 2008, 288 p. 
15. Gahramanov N.F., Mammadov N.S., Balayev V.A. Numerical computation methods in management, S: "Sumgayit", 2007, 160 p.

16. math.volchenko.com/Lectures/Interpol.pdf

17. Khanova A.A. Functions interpolation. Methodical manual for students of the Institute of Information Technologies and Communications, Astrakhan, 2001, 22 p.

18. Demyanov V.V., Saveliev E.A. Geostatistics: Theory and Practice, M.: Science, 2010, 327 p.

19. Geostatistical analysis. User's Guide, "ESRI Ltd", 2001, 278 p.

20. Aliyev E.M., Abbasov S.A., Sultanov E.T. Geographic database development to manage water resources // Problems of Information Technology, 2015, No1, pp.79-88.

21. Aliyev E.M. GIS analysis of real estate based on high resolution aerospace data // Problems of Information Technology, 2015, No2, pp.76-87.

22. Suleymanov T.I., Aliyev E.M. The use of geographic information systems technology in the mapping of the roads of the south-eastern regions of the Greater Caucasus // News of National Aerospace Agency, Baku, 2009, No1 (12), pp.11-15.

23. Alaskarov E.R. object-oriented classification of landscape using LIDAR data // Journal of Qafqaz University, Mathematics and Computer Science, Baku, 2013, No2 (1), pp.189-198.

24. static.bsu.az/w13/pages/2008-ci\%20ilin\%20hesabat\%C4\%B1.pdf

25. Aliyev E.M., Alaskarov E.R. Application of LIDAR data and geographic information technologies in the emergency situations of natural disasters // Problems of Information Technology, 2014, No2, pp.75-85.

26. Aliyev E.M. The study of the dynamics of the real estate market based on geographic information technologies and remote sensing data // Problems of Information Technology, 2012, No 1, pp. 61-69.

27. Aghayev A.T., Rustamov R.B. Remote Sensing and GIS / Geodatabase in river flood mapping // Journal of Surveying and Mapping Engineering, 2015, vol.3 no.1, pp.1-11.

28. Rajabov R.F. Mapping and analysis of turbidity in rivers of the Middle Kura depression // News of Baku University (natural sciences series), 2013, No2, pp.209-216.

29. Mutallibova S.F., Shabanov J.A. Evaluation and mapping of soil and vegetation of southern slope of the Greater Caucasus with aerospace methods // News of Baku University (natural sciences series), 2013, No3, pp.166-173.

30. Bayramov I.U. GIS in regional planning projects on the territory of the Republic of Azerbaijan // ARCREVIEW, Moscow, 2013, No2 (65), pp.10-12.

31. dataplus.ru/news/arcreview/detail.php?ID=1794\&SECTION_ID=48

32. Alyev E.M. Creating an electronic database for managing spatial objects based on orthophotos and GIS // ARCREVIEW (GIS in CIS), Moscow, 2013, No 2 (65), pp.13-14.

33. Mehbaliyev M.M. The morphometric study of the landscape of Zagatala reserve using GIS for the tourism development // ARCREVIEW, Moscow, 2010, No 1 (52), pp.14-15.

34. www.scribd.com/doc/272711909/Ismat-Baxishov

35. Sadullayev R.R., Aliyev E.M. The study of possibilities of ecotourism with the use remote sensing and GIS technologies // Works of the Azerbaijan Society of Geographical, 2011, XVII Volume, pp.443-450.

36. Mammadov R.M. Landscape Planning of Azerbaijan, Baku, 2009, p.142.

37. lup.lub.lu.se/luur/download?func $=$ downloadFile \&recordOId $=3558941 \&$ fileOId $=3558942$ 